\title{
Interplay Between Gravity and Quintessence: A Set of New GR Solutions
}

\author{
Arthur D. Chernin \\ Sternberg Astronomical Institute, Moscow University, Moscow, 119899, Russia, \\ Astronomy Division, Oulu University, Oulu, 90401, Finland, \\ and Tuorla Observatory, University of Turku, Piikkiö, 21 500, Finland \\ David I. Santiago \\ Department of Physics, Stanford University, Stanford, CA 940305, USA \\ Alexander S. Silbergleit \\ Gravity Probe B, W.W.Hansen Experimental Physics Laboratory, \\ Stanford University, Stanford, CA 940305-4085, USA
}

\begin{abstract}
A set of new exact analytical General Relativity (GR) solutions with time-dependent and spatially inhomogeneous quintessence demonstrate 1) a static non-empty spacetime with a horizon-type singular surface; 2) time-dependent spatially homogeneous 'spheres' which are completely different in geometry from the Friedmann isotropic models; 3) infinitely strong anti-gravity at a 'true' singularity where the density is infinitely large. It is also found that 4) the GR solutions allow for an extreme 'density-free' form of energy that can generate regular space-time geometries.

PACS numbers: 98.80.Cq, 04.25.Dm, 04.60-m
\end{abstract}




\section{Introduction. -}

The idea of quintessence (Q) as a dynamic, time-dependent and spatially inhomogeneous energy with negative pressure-to-density ratio $(p=w \rho,-1 \leq w<0)[1]$ provides new 'degrees of freedom' in cosmology $[1,2]$ and extends the variety of modern field models to include extreme forms of energy $[1,3]$. It may also stimulate a better understanding of the fundamental problem of interplay between gravity and the known field theories (for a review of this problem, see [4]).

The equation of state with $w=-1$ represents vacuum-type quintessence (VQ) which is phenomenologically described by the cosmological constant [5]; VQ is known to induce the dynamic effect of cosmological acceleration, if its density is positive. Recent data on the cosmological distribution of distant SN Ia [6] (as well as the evidence comming from the cosmic age, large scale structure, and cosmic microwave background anisotropy combined with the cluster dynamics) indicate that the observed cosmological expansion is indeed accelerating, most probably. The physical reason for the cosmic acceleration may generally be attributed not onlty to $\mathrm{VQ}$, but to any form of Q with $w<-1 / 3, \rho>0$ or $w>-1 / 3, \rho<0$, as seen from the Friedmann equations. In the original paper [1], positive energy for $\mathrm{Q}$ is preferred; in contrast with that, the whole range $-\infty<\rho<+\infty$ is considered below, for completeness and to examine whether any restrictions on the sign of energy could come from GR, if $w=$ const. Note that the "energy dominance condition', $\rho+p>0$, which is not met in the basic case of VQ, $w=-1$, is not satisfied also for some other forms of energy discussed below.

The special case $w=-1 / 3$ represents the only form of energy (with the linear equation of state) that can generate gravity with zero acceleration or deceleration effect in an isotropic universe, regardless the sign of the energy density. Gravity of this origin reveals itself only in the curvature of the four-dimensional space-time, it has no Newtonian analogs (unlike all other forms of $\mathrm{Q}$, including VQ), and its nature is completely due to General Relativity (GR) physics. Historically, Q with $w=-1 / 3$ was the first energy component that appeared in GR cosmology: in the Einstein static cosmological solution of 1917 the dynamic balance of anti-gravity of the cosmological constant and gravity of pressure-free matter gives rise to an effective equation of state with $w=-1 / 3$. 
This special type of $\mathrm{Q}$ with $w=-1 / 3$ may be called Einstein quintessence (EQ).

In the present Letter, some special properties of gravity produced by EQ, as well as by other extreme forms of $\mathrm{Q}$ in static and time-dependent, spatially homogeneous and inhomogeneous space-times are studied by means of a set of new exact analytical GR solutions. The metric of the solutions has 3D spherical symmetry,

$$
d s^{2}=A(r, t) d t^{2}-B(r, t) d \Omega^{2}-C(r, t) d r^{2}
$$

the componets of the metric tensor $A(r, t), B(r, t), C(r, t)$ are functions of the radial coordinate $r$ and time $t$, and $d \Omega^{2}=\sin ^{2} \theta d \varphi^{2}+d \theta^{2}$.

Four major new results are reported in the Letter. 1) The interplay between gravity and $\mathrm{Q}$ is able of creating a static non-empty space-time with a horizon-type singular surface; 2) This interplay is also revealed in the formation of time-dependent spatially homogeneous 'spheres' which are completely different from the Friedmann isotropic models and have no analogs in Newtonian gravity; 3) Q can induce infinitely strong anti-gravity at a 'true' singularity where the density is infinitely large; 4) GR allows for an extreme 'density-free' form of energy that can generate regular space-time geometries. We use the units with $G=c=1$.

\section{Solution $S \pm$.}

All 'static' [no dependence on $t$ in the metric coefficients (1)] solutions to Einstein's equations (see, for instance, [7]) with EQ equation of state $(w=-1 / 3)$ are given by

$$
B(r)=r^{2}, \quad C=\frac{r^{4}}{a^{2}} \frac{\left(A^{\prime}\right)^{2}}{A}, \quad 8 \pi \rho=\frac{3 a^{2}}{r^{5}} \frac{A}{\left(A^{\prime}\right)^{2}}\left(\frac{A^{\prime \prime}}{A^{\prime}}+\frac{2}{r}\right),
$$

where $A(r)$ is determined from a quadratic equation

$$
k A^{2}+\frac{2}{a^{2}} A+b+\frac{1}{r^{2}}=0
$$

with three arbitrary constants $k, b$ and $a^{2}>0$. For $k \neq 0$ the two $(\mathrm{S} \pm$ ) solutions thus are

$$
\begin{gathered}
A(r)=\frac{1}{k a^{2}}[-1 \pm \sqrt{K(r)}], \quad C(r)=\frac{-1 \mp \sqrt{K(r)}}{\left(b r^{2}+1\right) K(r)} \\
8 \pi \rho(r)=-\frac{3\left(1-k a^{4} b\right)}{k a^{4}}[-1 \pm \sqrt{K(r)}], \quad K(r)=1-k a^{4}\left(b+1 / r^{2}\right) .
\end{gathered}
$$


To describe the parameter cases, it is convenient to introduce $q \equiv 1-k a^{4} b$, so that $K(r)=q-k a^{4} / r^{2}$. Evidently, $\sqrt{K(r)}$ should be positive at least for some interval of $r$, which leads to the following:

A) For $q \leq 0, k>0$ there are no (physical) solutions

B) For $q<0, k<0$ we have "interior" solutions, $r^{2} \leq k a^{4} / q$; $\mathrm{G}+$ does not have a horizon for $q<-1 / 2$, its horizon coincides with the boundary $r^{2} \geq 2|k| a^{4}$ for $q=-1 / 2$, there is a horizon inside the spacetime for $-1 / 2<q<0$; apparently, S- always has no horizon.

C) For $q=0, \rho=0, k<0 \mathrm{~S}+$ is the Schwarzshild solution, $\mathrm{S}$ - is not physical because it is also Schwarzshild but with the negative central mass.

D) For $q>0, k<0$ both solutions are "global" (spacetime extends to any $r$ ), S+ has a horizon.

E) For $q>0, k>0$ both are "exterior" solutions, $r^{2} \geq k a^{4} / q$, S+ has no horizon for $0<q<1 / 2$, its horizon coincides with the boundary $r^{2} \geq 2 k a^{4}$ for $q=1 / 2$, there is a horizon inside the spacetime for $q>1 / 2$.

Note that whenever $r=0$ is within the spacetime, solutions $\mathrm{S} \pm$ have there a true (non-coordinate) singularity: both the Ricci curvature and the density are infinite at $r=0$. For $\mathrm{S}-$ this is a naked singularity, with no horizon around it. Note also that in the lomit $k \rightarrow 0 \mathrm{~S}+$ goes to the solution $\mathrm{S}-1$, which is obtained from Eq.(3) with $k=0$ and is discussed immediately below, while S- turns to infinity.

\section{Solution S-1. -}

If $k=0$ in Eq.(3), we have a particular solution for EQ of an especially simple form:

$$
\begin{gathered}
A(r)=A_{0}\left(1-\frac{1}{\alpha r^{2}}\right), \quad B(r)=r^{2}, C(r)=\frac{2}{\alpha r^{2}}\left(1-\frac{1}{\alpha r^{2}}\right)^{-1} ; \\
8 \pi \rho=\frac{3}{2} \alpha\left(\frac{1}{\alpha r^{2}}-1\right)
\end{gathered}
$$

where $\alpha=-b$ and $A_{0}=a^{2} \alpha / 2$. To keep the proper signature of the metric, we require $A_{0}>0$, so that $\alpha>0$; without loss of generality, we set $A_{0}=1$, i. e., $a^{2}=2 / \alpha=$ $-2 / b>0$. Let us discuss this solution in more detail, setting, for simplicity, $\alpha=1$.

A) The space-time of the solution S-1 has a horizon at $r=1$ where the components of the metric tensor $g_{00}=A(r)$ and $g_{11}=-C(r)$ change their signs. The horizon separates 
(or connects) a static spatially inhomogeneous exterior space (E-space) at $r>1$ and a 'hidden' interior object (I-object) at $r<1$. These two regions are similar to R- and T-regions, respectively, as described by Novikov [8] for the Schwarzschild solution. The true singularity with the infinite Ricci curvature and density is at $r=0$. The density in S-1 may be regarded as consisting of two components with the same equation of state: one is uniform and negative, $8 \pi \rho_{-}=-3 / 2<0$, the other is nonuniform ('isothermal' law) and positive, $8 \pi \rho_{+}(r)=3 / 2 r^{2}>0$. The first one dominates the E-space, and the other one dominates the I-object; the total density changes its sign at the horizon.

B) The 'energy equation' introduced for the metric of Eq.(1) by Lemaitre (see Ref. [5]) may be used to find an analog of the Newtonian gravitational potential as one of the physical characteristics of gravity produced by EQ in S-1. The equation has a form of energy conservation relation, $\frac{1}{2}\left(d B^{1 / 2} / d t\right)^{2}-m(r, t) / r=E$, where $E=C^{-1}\left(d B^{1 / 2} / d r\right)^{2}-1$ and $m(r, t)$ is the Newtonian analog of gravitating mass. In S-1, the Newtonian mass proves to be $m(r)=(3 / 4) r\left[1-(1 / 3) r^{2}\right]$, and the Newtonian potential $u(r)=-m / r=$ $-(3 / 4)\left[1-(1 / 3) r^{2}\right]$ describes 'the Newtonian component' of gravity produced by EQ in S-1. The potential $u$ is finite at the true singularity $[u(0)=-3 / 4]$ (unlike that in Schwarzchild solution) and goes to $+\infty$ at spatial infinity where density becomes constant (see below). On the singular surface, $m(1)=1 / 2$, and so $2|u(1)|=1$ (like that in the Schwarzschild solution).

C) EQ gravity in S-1 is not reduced to the Newtonian gravity completely; in particular, the dynamic effect of acceleration is essentially different in S-1 from what may be produced by the Newtonian potential $u$. The total accelerating effect can be evaluated in terms of the effective potential, $U(r)$, which may be derived using the fact that S-1 describes EQ in a state of hydrostatic equilibrium: its pressure and selfgravity are balanced in the E-space. The pressure gradient produces radial acceleration $F_{p}=-(d p / d r)(\rho+p)^{-1}=\left[8 \pi\left(r^{3}-r\right)\right]^{-1}$, which is positive. So the gravity acceleration is $F=-F_{p}=-\left[8 \pi\left(r^{3}-r\right)\right]^{-1} ;$ it is negative, and therefore gravity is attracting. The effective gravitational potential responsible for this attraction is $U=(1 / 2) \ln \left[r^{2} /\left(r^{2}-1\right)\right]$. D) S-1 can be rewritten in the E-space as

$$
d s^{2}=\tanh ^{2}(\chi / \sqrt{2}) d t^{2}-\cosh ^{2}(\chi / \sqrt{2}) d \Omega^{2}-d \chi^{2}
$$




$$
8 \pi \rho=-\frac{3}{2} \tanh (\chi /(\sqrt{2})
$$

with the new spatial coordinate $\chi$ related to $r$ by $r=\cosh (\chi /(\sqrt{2})$; its range is from zero to infinity. The density decreases monotonically from zero at $\chi=0$ to $-3 / 2$ at $\chi=\infty$. This form of S-1 may possibly be considered both in relation to and independently of the I-object. In the limit $r \rightarrow \infty$, where density is spatially homogeneous, the metric of S-1 takes the form $d s^{2}=d t^{2}-r^{2} d \Omega^{2}-2 r^{-2} d r^{2}$, or $d s^{2}=d t^{2}-\exp (\sqrt{2} \chi) d \Omega^{2}-d \chi^{2}$. Since the general static solution for EQ in the isotropic 3-space has the form $d s^{2}=$ $d t^{2}-r^{2} d \Omega^{2}-\left(1-k r^{2} / a_{0}^{2}\right)^{-1} d r^{2}, 8 \pi \rho=-24 \pi p=3 k / a_{0}^{2}, \quad(k=1,0,-1$ is the sign of $3-$ curvature, $a_{0}$ is an arbitrary scalling constant), one can see that the metric of Eqs. $(2,4)$ reduces to the isotropic metric with $k=-1$ and $a_{0}=1 / 2$ in the limit $r \rightarrow \infty$. For comparison: $k=1, a_{0}=1$ in the Einstein static solution.

E) Since $A(r)$ and $C(r)$ in S-1 change their signs at the singular surface, the signature of the metric of the I-object is $(---+)$. This means that the coordinate $r$ becomes timelike, and the coordinate $t$ becomes space-like inside the I-object. The same is true for the Schwarzschild space-time ([8]). Because of that, the density of the I-object depends only on the time-like coordinate, thus it is spatially homogeneous. However, in contrast with the Schwarzschild space-time, a formal replacement of $g_{00}$ with $g_{11}$ and vice versa, which transforms the signature of the I-object to the 'ordinary' type (+ - - ), does not work for S-1, because the metric resulting from Eq.(2) after this transformation is not a solution to the GR equations.

\section{Solution S-2.}

GR equations for the same metric of Eq.(1) allow for the following time-dependent particular solution:

$$
\begin{gathered}
A(t)=\frac{1}{4}\left(n^{2}-4\right)=\text { const }>0, \quad B(t)=t^{2}, \quad C(t)=t^{n}, \\
8 \pi \rho=\frac{n(n+4)}{n^{2}-4} \frac{1}{t^{2}}, \quad p=w \rho, \quad n=-\frac{4 w}{1+w}=\text { const. }
\end{gathered}
$$

The parameter range of this solution apparently consists of two parts: $n>2(-1<$ $w<-1 / 3)$, and $n<-2(|w|>1$; this is not $\mathrm{Q}$ as defined above). Note that both VQ $(w=-1)$ and EQ $(w=-1 / 3)$ are outside this range. 
A) The most striking feature of this solution (S-2) is that $A, C$ and even $B$ do not depend on $r$, and are functions of the time only. One may see here a similarity to the I-object of S-1 whose metric also depends on the time-like coordinate only. A more close similarity may be recognized with the 'T-sphere' found by Ruban [9] for pressure-free matter and the same as in S-2 symmetry of 3 -space with $B=B(t)$. The density and pressure are spatially homogeneous (functions of the time only) in both the S-2 and Ruban's T-sphere. Time $t$ varies in S-2 from $-\infty$ to $+\infty$, and the density varies from zero at $t \rightarrow \pm \infty$ to infinity in (true) singularity $t=0$ which has the same character as that in isotropic cosmological models. However, there are no coordinate transformations that could reduce S-2 and Ruban's T-sphere to the FRW metric with the isotropic 3-space.

B) Unlike the Friedmann solutions, gravity in S-2 and Ruban's T-sphere does not have any Newtonian analogs. Rather, the space-time of this special type has common features with anisotropic spatially homogeneous cosmological models (cf. [9]).

C) The pressure, $p=-n^{2} /\left[8 \pi\left(n^{2}-4\right) t^{2}\right]$, is negative in S-2, while the density may be either positive or negative. The density is positive if $w$ is in 'the $\mathrm{Q}$ range' $-1<w<-1 / 3$ $(n>2)$. (Note that in the isotropic 3 -space $\mathrm{Q}$ with $w$ in this range produces positive acceleration, see Introduction.) The density is also positive for $w>1$ (i. e., $-4<n<2$ ), but it is negative when $w<-1(n<-4)$.

D) Density turns to zero for $w=\infty(n=-4)$. This case describes a form of energy with $\rho=0, p<0$, which is perhaps the most extreme form of Q. As we see, GR does not exclude the equation of state with $w=\infty$, and gives a regular solution for it, $A(r)=3, \quad B(r)=t^{2} \quad C=t^{-4}$. 'Density-free' energy may also be in a state of hydrostatic equilibrium (see below).

Solution S-3.

A power law particular static solution, which is a counterpart to S-2, is also allowed by GR equations for the metric of Eq.(1):

$$
\begin{gathered}
A(r)=r^{n}, \quad B(r)=r^{2}, \quad C(r)=\frac{1}{4}\left(4+4 n-n^{2}\right)=\text { const }>0, \\
8 \pi \rho=\frac{n(4-n)}{4+4 n-n^{2}} \frac{1}{r^{2}}, \quad p=w \rho, \quad n=\frac{4 w}{1+w} .
\end{gathered}
$$

The parameter range here looks even more peculiar: $-2(\sqrt{2}-1)<n<2(\sqrt{2}+1)$, which 
corresponds to the two intervals for the values of $w, w>-(\sqrt{2}-1) /(\sqrt{2}+1)>-1 / 3$ and $w<-1$. Neither VQ, nor EQ are within this range, and the solution relates to $\mathrm{Q}$ only when $-(\sqrt{2}-1) /(\sqrt{2}+1)<w<0$.

A) Similar to S-1 and S-2, solution S-3 depends on one coordinate only, and the true singularity of all the three solutions is at the origin of this coordinate. The positive component of density in S-1, and the total densities in S-2 and S-3 follow the inverse square law. Both S-1 and S-3 describe Q in a state of hydrostatic equilibrium.

B) The pressure, $p=n^{2} /\left[8 \pi\left(4+4 n-n^{2}\right) r^{2}\right]$, is positive in S-3 for any $n \neq 0$. At $n=0$ the pressure and density are both identical zeros. In this case, $A=1, C=1$, and the metric of S-3 turns to the Lorentz metric of the empty space-time. The density in S-3 is positive for $0<n<4$ and negative otherwise.

C) The density vanishes also for $n=4, w= \pm \infty$. This is the 'density-free' $(\rho=0, p>0)$ form of energy, and S-3 (same as S-2 above) gives a regular metric for this case: $A=$ $1, B(r)=r^{2}, C(r)=r^{4}$. The fact that this form of energy is in the state of hydrostatic equilibrium shows that 'density-free' energy has its inertial mass (per unit volume) $\rho_{i}=p$ which is equal to its passive gravitational mass, and that the active mass is positive.

D) For $n \neq 0$, the sign of the active gravitating mass depends on the sign of $n$. If $n$ is positive, the mass is positive; but if $n$ is negative (and thus $w$ is negative), the active mass is negative also. In terms of Newtonian physics, the negative mass produces a positive acceleration, which goes to infinity as $r \rightarrow 0$, and so anti-gravity is infinitely strong at the singularity. Whether it is capable, under these conditions, of producing 'auto-emission' of particles from the singularity (where the density is infinite), and/or enhance quantum evaporation of particles from the singularity, should be discussed separately.

E) S-3 describes hydrostatic equilibrium of not only $\mathrm{Q}$ with negative $w$, but also, for instance, of ultra-relativistic fluid $(w=1 / 3, n=1, C=7 / 4$,$) and of Zeldovich ultra-stiff$ fluid $(w=1, n=2, C=2)$.

[1] R.R. Caldwell, R. Dave, P.J. Steinhard, Phys.Rev.Lett. 80, 1582 (1998)

[2] R.R. Caldwell, R. Dave, P.J. Steinhard, Ap. Space Sci. 261, 303 (1998); L. 
Wang, P.J. Steinhard, Astrophys. J. 508, 483 (1998); C.-P. Ma, R.R. Caldwell, P. Bode, L. Wang, Astrophys. J. 521, L1 (1999); A.R. Cooray, D. Huterer, Astrophys. J. 513, L95 (1999); J.S. Alcaniz, J.A.S. Lima, Astron. Astrophys. 349, 729 (1999); I.S. Zlatev, P.J. Steinhard, Phys. Lett. B 459, 570 (1999); L. Hui, Astrophys. J. 519, L9 (1999); I. Zlatev, Wang L., P.J. Steinhard, Phys. Rev. Lett. 82, 896 (1999); P.J.E. Peebles, A. Vilenkin, Phys. Rev. D 590, 811 (1999); M. Giovannini, Phys. Rev. D 601, 277 (1999); L. Wang, R.R. Caldwell, J.P. Ostriker, P.J. Steinhard, Astrophys. J. 550, 17 (200); G. Efstathiou, MN RAS 310, 842 (2000); P.F. González-Díaz, Phys. Lett. B 481, 353 (2000); J.D. Barrow, R. Bean, J. Magueijo, MN RAS 316, L41 (2000)

[3] S.M. Carroll, Phys. Rev. Lett. 81, 3067 (1998); S.M. Barr, Phys. Lett. B 454, 92 (1999); R.S. Kalyana, Phys. Lett. B 457, 268 (1999); Ch. Kolda, D.H. Lyth, Phys. Lett. B 459, 570 (1999); P. Binétruy, Phys. Rev. D 600, 80 (1999); R. Horvat, Mod. Phys. Lett. A 14, 2245 (1999); T. Chiba, Phys. Rev. D. 601, 4634 (1999); P.H. Brax, J. Martin, Phys. Lett. B 468, 40 (1999); A.B. Kaganovich, Nuc. Phys. B Proc.Suppl. 87, 496 (1999); O. Bertolami, P.J. Martins, Phys. Rev. D 610, 7 (2000); Y. Nomura, T. Watari, T. Yanagida, Phys. Lett. B 484, 103 (2000); S.C.C. Ng, Phys. Lett. B 485, 1 (2000); Dynmikova I. G. Phys. Lett. B472, 33 (2000); A. Hebecker, C. Wetterich, Phys. Rev. Lett. 85, 3339 (2000); N. Arkani-Hamed, L.J. Hall, C. Colda, H. Murayama, Phys. Rev. Lett. 85, 4434 (2000); C. Armendariz-Picon, V. Mukhanov, P.J. Steinhard, Phys. Rev. Lett. 85, 4438 (2000)

[4] S. Weinberg, Rev. Mod. Phys. 61, 1 (1989)

[5] G. Lemaitre, Rev. Mod. Phys. 21, 357 (1949); E.B. Gliner, JETP 22, 378 (1966); E.B. Gliner, Sov. Phys. Doklady 6, 559 (1970).

[6] A.G. Riess et al., Astron. J. 116, 1009 (1998); S. Perlmuter et al., Astrophys. J. 517, 565 (1999); J. Cohn, astro-ph/9807128; S. Carol, astro-ph/0004075

[7] L.D. Landau, E.M. Lifshitz, The Classical Field Theory, Pergamon Press, LondonParis (1959)

[8] I.D. Novikov, Comm. Sternberg Astron. Inst. 132, 43 (1963)

[9] V.A. Ruban, JETP 29, 1027 (1969) 